\section{Puesta al día: carcinoma paratiroideo}

\author{
MARCELA BARBERÁN ${ }^{1,2}$, CLAUDIA CAMPUSANO $^{3}$, \\ PATRICIO SALMAN ${ }^{4}$, PAMELA TREJO ${ }^{5}$, \\ ANGÉLICA SILVA-FIGUEROA ${ }^{6,7}$ SANDRA RIVERA $^{8}$, \\ PABLO FLORENZANO ${ }^{9}$, SOLĖDAD VELASCO $^{4}$, \\ FRANCISCA ILLANES ${ }^{4}$, PATRICIO TRINCADO ${ }^{1}$, \\ JOSÉ CANESSA ${ }^{10}$, ANTONIETA SOLAR ${ }^{11}$, \\ MARCELA MORENO ${ }^{3,12}$, DANIELA EUGENIN ${ }^{2,3}$, \\ BEATRIZ JIMÉNEZ ${ }^{13}$, PATRICIA ARROYO ${ }^{14}$
}

\section{An update on parathyroid carcinoma}

Parathyroid carcinoma is a rare malignant disease that presents as a sporadic or familial primary hyperparathyroidism (PHP). The latter is associated with some genetic syndromes. It occurs with equal frequency in both sexes, unlike PHP caused by parathyroid adenoma that is more common in women. It should be suspected in cases of severe hypercalcemia, with high parathyroid hormone levels and a palpable cervical mass. Given the difficulty in distinguishing between parathyroid carcinoma and adenoma prior to the surgery, the diagnosis is often made after parathyroidectomy. The only curative treatment is complete surgical resection with oncologic block resection of the primary tumor to ensure free margins. Adjuvant therapies with chemotherapy or radiation therapy do not modify overall or disease-free survival. Recurrences are common and re-operation of resectable recurrent disease is recommended. The palliative treatment of symptomatic hypercalcemia is crucial in persistent or recurrent disease after surgery since morbidity and mortality are more associated with hypercalcemia than with tumor burden.

(Rev Med Chile 2021; 149: 399-408)

Key words: Diagnosis; Hypercalcemia; Hyperparathyroidism; Parathyroid Neoplasms.

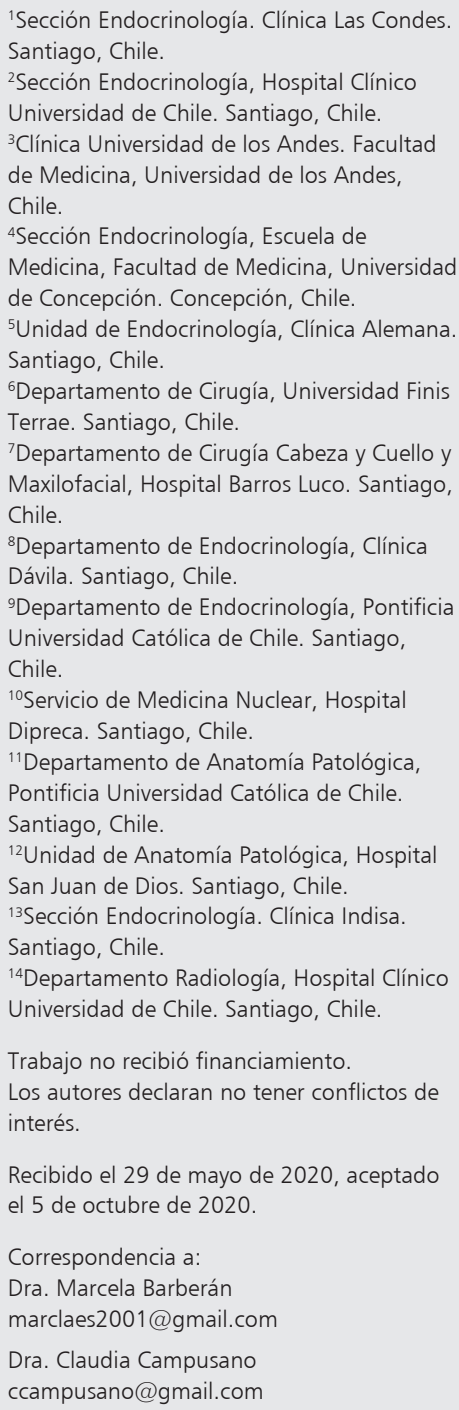

$\mathrm{E}$ 1 cáncer de paratiroides $(\mathrm{CP})$ es un tumor endocrino infrecuente que se presenta, habitualmente, como hiperparatiroidismo primario (HPP) esporádico, pero puede también ocurrir en forma familiar. Es altamente agresivo, pero de curso indolente. Se estima una incidencia anual inferior a 1 caso por millón de habitantes. Ocurre a cualquier edad, siendo más frecuente en la quinta década de la vida, sin diferencias en ambos sexos. Más del 90\% de los casos se presenta como un HPP, pero constituyen menos de 5\% de estos pacientes.
A pesar de recientes desarrollos bioquímicos, radiológicos y moleculares, el $\mathrm{CP}$ sigue siendo una enfermedad difícil de reconocer clínica e histológicamente, lo que plantea un desafío, ya que es crítico un diagnóstico precoz para que el tratamiento pueda ser exitoso ${ }^{1}$.

\section{Presentación clínica}

A diferencia de los HPP benignos, la mayoría de los cánceres son hormonalmente muy activos 
$y$, frecuentemente, se presentan como hipercalcemia grave, que se manifiesta antes de los síntomas provocados por la invasión local o regional del tumor $^{2}$. Menos de $10 \%$ de los CP son no funcionantes $y$, generalmente, se diagnostican en etapas más avanzadas con síntomas relacionados al efecto de masa ${ }^{2,3}$.

En el CP funcionante, el compromiso renal y esquelético son relevantes y son concomitantes en $50 \%$. El compromiso esquelético se observa en $90 \%$ de los pacientes y se manifiesta como dolor óseo, osteoporosis, osteítis fibrosa quística y fracturas patológicas. La nefrolitiasis o insuficiencia renal ocurre en el $80 \%$ de los $\operatorname{casos}^{2,4}$. Las complicaciones digestivas incluyen constipación, úlceras pépticas y pancreatitis, y los síntomas neuropsiquiátricos, compromiso de conciencia y depresión.

Las crisis hipercalcémicas ocurren en 7 a 12\% de los CP y se presentan con calcemias mayores a $14 \mathrm{mg} / \mathrm{dl}$ y presentan oligoanuria y manifestaciones neurológicas de magnitud variable ${ }^{1,4}$.

En el examen físico, se observa una masa cervical palpable en 40 a $70 \%$ de los casos. Este hallazgo, en presencia de HPP, debe desencadenar la sospecha de CP. Los sitios más frecuentes de metástasis son los ganglios linfáticos regionales (15-20\%), pulmón, hígado y hueso (1\%).

\section{Laboratorio}

Los niveles séricos de hormona paratiroidea $(\mathrm{PTH})$ pueden ser 10 a 15 veces mayores que lo normal. En un estudio reciente, un nivel de PTH 10 veces o más que el límite superior normal tenía un valor predictivo positivo de $81 \%$ para $\mathrm{CP}^{3}$.

La calcemia promedio es más alta que en los HPP benignos y suelen ser $14 \mathrm{mg} / \mathrm{dl}^{2}$. Los niveles de fosfatasas alcalinas y subunidades $\alpha$ y $\beta$ de gonadotropina coriónica humana pueden ser mayores en pacientes con carcinoma (Tabla 1).

\section{Formas familiares y recomendaciones de estudio genético}

El CP se ha asociado a mutaciones del gen cell division cycle protein 73 (CDC73, también conocido como HRPT2). Este gen codifica para la proteína parafibromina que tiene una función supresora de tumores. Mutaciones de este gen se asocian a hiperparatiroidismo asociado a tumor mandibular (HPT-JT), a cáncer de paratiroides aislado y a HPP aislado familiar.
El HPT-JT es una enfermedad hereditaria autosómica dominante causada en $75 \%$ de los casos por mutaciones germinales inactivantes de CDC73. Se caracteriza por hiperparatiroidismo hereditario asociado a fibromas osificantes en los maxilares y tumores paratiroideos funcionantes, de los cuales, $15 \%$ corresponden a CP. Además, pueden presentar tumores renales y uterinos.

En el cáncer esporádico de paratiroides se han encontrado mutaciones somáticas de CDC73 entre 40 y $100 \%$ de los casos, y germinales en aproximadamente $30 \%$. La identificación de pacientes con mutaciones germinales permite detectar a los familiares portadores para realizar un diagnóstico temprano. Por esta razón, se ha sugerido el estudio de $C D C 73$ en todos los pacientes con CP aparentemente esporádico ${ }^{6,7}$.

El hiperparatiroidismo aislado familiar se define por la presencia de la enfermedad en dos o más familiares de primer grado, sin elementos clínicos de otros síndromes genéticos. Se ha relacionado a mutaciones activantes del gen GMC2 (glial cells missing transcription factor 2) pero se han comunicado familias con mutaciones germinales en genes MEN1 (neoplasia endocrina múltiple tipo 1), CDC73 y CASR (receptor sensor del calcio) ${ }^{8,9}$.

El gen $M E N 1$ codifica para la proteína menina que también es supresora de tumor. Mutaciones inactivantes en este gen son causantes de neopla-

\section{Tabla 1. Criterios de sospecha clínica de cáncer paratiroideo}

Edad de 44 a 54 años
Calcemia superior a $14 \mathrm{mg} / \mathrm{dl}$
PTH 10 a 15 veces mayor al máximo normal
Crisis hipercalcémica
Masa palpable del cuello (lesión $>3 \mathrm{~cm}$ )
Enfermedad ósea metabólica
Enfermedad renal
Pancreatitis
Antecedentes personales o familiares de hipercalcemia o
síndromes genéticos (HPT-JT, MEN)
Tumor de la mandíbula concomitante
Infiltración o calcificaciones en ultrasonido de cuello
Sospecha de enfermedad metastásica en SestaMIBI o TAC


sias endocrinas múltiple que corresponde a un desorden autosómico dominante que produce HPP, tumores hipofisiarios y pancreáticos. La presencia de hiperparatiroidismo suele ser la primera manifestación, habitualmente con compromiso multiglandular y con mayor prevalencia de glándulas paratiroideas ectópicas. Es muy infrecuente que estos individuos presenten $\mathrm{CP}$, con una prevalencia de 0,28 a $2 \%$ de los casos.

En suma, es altamente aconsejable realizar estudio genético para buscar mutaciones de CDC73 en pacientes con cáncer de paratiroides aislado $^{5}$, hiperparatiroidismo asociado a tumor mandibular, hiperparatiroidismo primario aislado familiar, para mejor caracterización del paciente y poder identificar mutaciones germinales que permitan el diagnóstico temprano de familiares portadores (Tabla 2).

\section{Estudio con imágenes}

La técnica imagenológica inicial, luego de la sospecha clínica del CP, es la ecografía, por su amplia disponibilidad y bajo costo. Los adenomas paratiroideos normosituados son nódulos extratiroideos, generalmente sólidos, hipoecogénicos con respecto al parénquima tiroideo y vascularizados. En cambio, las características ecográficas que sugieren malignidad son tamaño aumentado (sobre $203 \mathrm{~cm}$ en diámetro mayor), contornos lobulados e invasión de tejidos vecinos (tiroides, músculos, vena yugular) ${ }^{10}$. El hallazgo de adenopatías es infrecuente al diagnóstico, aunque su presencia es sugerente de malignidad. Otros hallazgos descritos con menor frecuencia y especificidad incluyen cambios quísticos y presencia de calcificaciones ${ }^{11}$. Su sensibilidad es de $80 \%{ }^{11,12}$. El rendimiento de estos exámenes aumenta al combinarlos con métodos de medicina nuclear, pero ninguno es útil para evaluar el grado de invasión local o a distancia ${ }^{13}$.

La técnica de medicina nuclear más usada es la cintigrafía con SestaMIBI (MIBI) marcado con Tecnecio ${ }^{99 m}$, con SPECT/CT (Single Photon Emission Computed Tomography acoplado a tomografía computada), con adquisición de imágenes tempranas y tardías (10 y $120 \mathrm{~min}$ ) posterior a inyección del trazador. Tiene la ventaja, sobre el ultrasonido, que visualiza el mediastino y el resto del cuello, lo que permite estudiar glándulas paratiroideas ectópicas ${ }^{14}$. Esta técnica tiene falsos positivos, relacionados con patología nodular, neoplásica e inflamatoria del tiroides. Los falsos negativos se relacionan con tamaño glandular pequeño y con hiperplasia multiglandular ${ }^{14}$.

El CP fue incluido en la $8^{\text {a }}$ edición de la clasificación TNM (tumor, adenopatías, metástasis) del año 2017 para etapificación ${ }^{15}$ (Tabla 5). Con este fin se ha propuesto la utilización de varias técnicas de imágenes como tomografía axial computada (TAC) y resonancia magnética (RM) que permiten evaluar la invasión local y a distancia y la aparición de recurrencias.

La TAC debe solicitarse en todos los pacientes en que se sospeche CP. Permite evaluar zonas más profundas del cuello y mediastino para determinar invasión a tejidos vecinos como esófago y tráquea y tener mejores referencias anatómicas para planear

Tabla 2. Situaciones clínicas en que debe considerarse el estudio genético

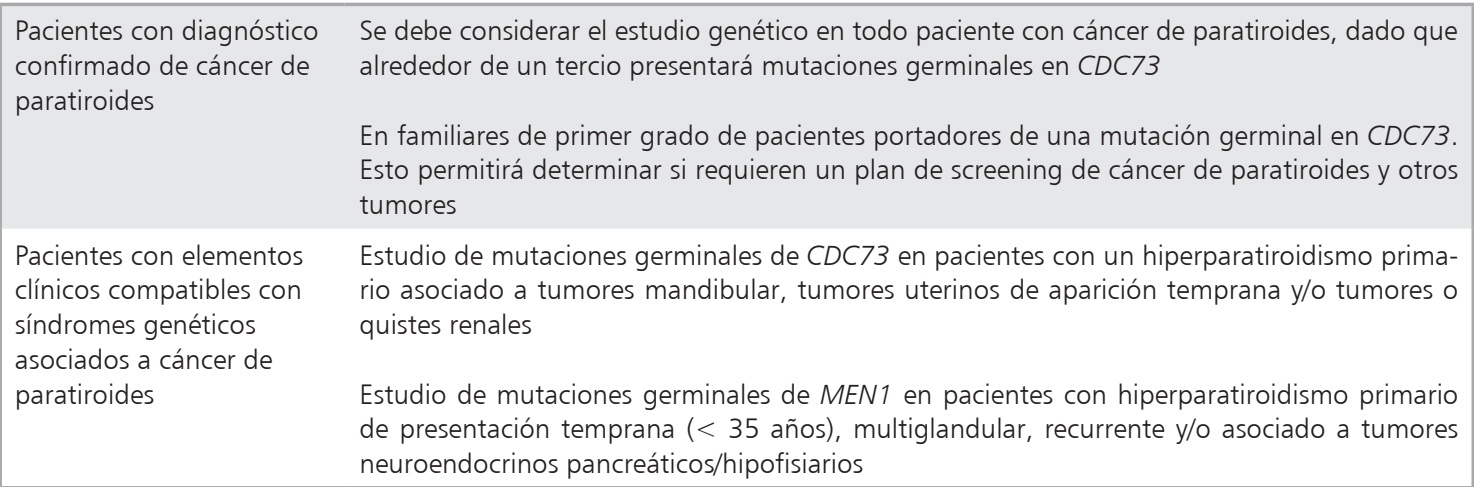

Se debe considerar el estudio genético en todo paciente con cáncer de paratiroides, dado que alrededor de un tercio presentará mutaciones germinales en CDC73

En familiares de primer grado de pacientes portadores de una mutación germinal en CDC73. Esto permitirá determinar si requieren un plan de screening de cáncer de paratiroides y otros tumores

Estudio de mutaciones germinales de CDC73 en pacientes con un hiperparatiroidismo primario asociado a tumores mandibular, tumores uterinos de aparición temprana y/o tumores o quistes renales

Estudio de mutaciones germinales de MEN1 en pacientes con hiperparatiroidismo primario de presentación temprana ( $<35$ años), multiglandular, recurrente y/o asociado a tumores neuroendocrinos pancreáticos/hipofisiarios

Estudio de mutaciones germinales de MEN1, CDC73 y CASR (receptor sensor de calcio) en hiperparatiroidismo familiar aislado. 
una cirugía. Las lesiones tumorales de CP en TAC son captantes de contraste.

La RM también contribuye a una mejor evaluación preoperatoria. Los adenomas paratiroideos generan una imagen de intensidad indeterminada en T1 e hiperintensa en T2 al administrar gadolinio, siendo las secuencias contrastadas realizadas con supresión de grasa, las más útiles para la identificación de lesiones paratiroideas ${ }^{14}$.

Entre las nuevas técnicas se encuentra la tomografía con emisión de positrones (PET) con tecnología híbrida con TAC o RM. De los diferentes trazadores, el más usado es la desoxiglucosa marcada con flúor ${ }^{18}$ ( $\left.\mathrm{F}^{18}-\mathrm{FDG}\right)$, aunque aplicado en etapificación del CP no ha mostrado superioridad sobre técnicas convencionales ${ }^{16}$. Puede ser más útil usar como trazador colina marcada con carbono ${ }^{11}$ o flúor ${ }^{18}\left(\mathrm{~F}^{18} \mathrm{CH}-\mathrm{PET}\right)$. Es un precursor de fosfatidilcolina, componente fosfolipídico de la membrana celular, y su metabolismo está aumentado en células neoplásicas de próstata y paratiroides. Tiene una sensibilidad de $91-95 \%$, pero es de mayor costo y menor disponibilidad ${ }^{17}$. En el infrecuente caso de CP no funcionante, SPECT MIBI no tiene indicación.

Otro aspecto del impacto de la hipersecreción de PTH es el gran aumento del recambio óseo, especialmente en hueso cortical. Puede existir osteítis fibrosa quística (tumores pardos, quistes óseos, etc.) que pueden ser confundidos con metástasis óseas osteoblásticas en la cintigrafía ósea convencional $^{18}$, por lo que debe tenerse presente en el diagnóstico diferencial.

\section{Anatomía patológica}

El CP puede originarse en cualquiera de las glándulas paratiroideas normotópicas o ectópicas, sin predilección por ningun sitio ${ }^{19}$.

Macroscópicamente, son de mayor tamaño que los adenomas, fluctuando entre 1,5 y $27 \mathrm{~g}$ con promedio de $6,7 \mathrm{~g}$, pudiendo ser encapsulados o de bordes infiltrativos. En el intraoperatorio suelen ser difíciles de disecar por su adherencia a tejidos adyacentes ${ }^{20}$. Al corte son firmes y pueden tener áreas de necrosis, calcificación y fibrosis. Histológicamente, el diagnóstico de carcinoma es difícil y debe hacerse bajo parámetros estrictos inequívocamente asociados a invasión de estructuras. Se han agrupado en criterios absolutos y criterios asociados a malignidad ${ }^{21}$ (Tabla 3 ). Para los casos en que no se encuentran todos los hallazgos para ser diagnosticados como malignos, deben ser designados como adenoma atípico.

\section{Tabla 3. Criterios de anatomía patológica}

\begin{tabular}{|c|c|}
\hline \multirow{5}{*}{$\begin{array}{l}\text { Criterios absolutos. } \\
\text { Basta solo uno de ellos } \\
\text { para el diagnóstico de } \\
\text { carcinoma }\end{array}$} & Invasión de tejidos blandos periparatiroideo \\
\hline & Invasión a órganos vecinos como tiroides, laringe, faringe, esófago, nervio laríngeo recurrente, etc. \\
\hline & Invasión perineural \\
\hline & $\begin{array}{l}\text { Invasión vascular: esta debe estar presente en los vasos de la cápsula o del tejido peritumoral; } \\
\text { las células tumorales deben estar adheridas a la pared del vaso total o parcialmente, asociadas a } \\
\text { fibrina y pueden o no estar cubiertas por células endoteliales }{ }^{13}\end{array}$ \\
\hline & $\begin{array}{l}\text { Presencia de metástasis con confirmación histológica. Los sitios más frecuentes son ganglios } \\
\text { linfáticos cervicales e hígado }{ }^{14}\end{array}$ \\
\hline \multirow{7}{*}{$\begin{array}{l}\text { Criterios asociados a } \\
\text { malignidad. } \\
\text { La presencia de más } \\
\text { de } 3 \text { de estos hechos } \\
\text { indica un alto riesgo } \\
\text { de comportamiento } \\
\text { maligno }^{12}\end{array}$} & Compromiso capsular sin extensión al tejido fibroadiposo periglandular \\
\hline & Más de 5 mitosis por 10 campos de aumento mayor \\
\hline & Amplias bandas de fibrosis intraglandulares, separando nódulos expansivos \\
\hline & Necrosis de coagulación, presente en un tercio de los carcinomas ${ }^{14}$ \\
\hline & $\begin{array}{l}\text { Crecimiento difuso de células dispuestas en sábanas con aumento de la relación núcleo citoplas- } \\
\text { mática }\end{array}$ \\
\hline & Atipía celular difusa \\
\hline & Macro nucléolos en la mayoría de las células \\
\hline
\end{tabular}

Para los casos en que no se encuentran todos los hallazgos para ser diagnosticados con riesgo de malignidad, deben ser designados como adenoma atípico. 
La inmunohistoquímica con PTH es útil para confirmar la naturaleza de los CP, ya sean funcionantes, no funcionantes o ectópicos. Los CP muestran frecuentemente, pero no siempre, pérdida de la expresión de parafibromina ${ }^{22,23}$.

La inmunotinción difusa para PGP9.5 (producto proteico del gen 9,5) suele ser positiva en $\mathrm{CP}$, con una sensibilidad de $78 \%$ y especificidad de $100 \%{ }^{24}$. El marcador de proliferación Ki 67 puede ayudar para la distinción entre CP y adenoma, el primero con un índice entre 6,1 y $8,4 \%$, el segundo entre 2,0 y $3,3 \%$; sin embargo, dada la superposición, no puede ser utilizado como elemento único para definir malignidad ${ }^{25-27}$; un índice sobre $5 \%$ sugiere carcinoma, pero un índice bajo no lo descarta. La expresión de galectina-3 es frecuente en el CP, $92 \%$ de los casos son positivos, y puede ayudar a distinguirlos de un adenoma, en que solo 3\% resultan positivos. La sobreexpresión de la ciclina D1 ha sido descrita en 91\% de los casos de los CP. Por último, la pérdida de la inmunorreactividad para la proteína RB (codificada por el gen supresor de tumor retinoblastoma) ha sido descrita en 20\% a 100\% de los CP y algunos autores sugieren que ese marcador ayuda a la distinción, sin embargo, no existe consenso. El uso de un panel que incluya al menos tres marcadores de entre los siguientes: Ki 67, E-cadherina, galectina-3, parafibromina y $\mathrm{RB}$, sería más útil para distinguir un carcinoma de un adenoma, que cada uno de estos marcadores por si $\operatorname{solos}^{28}$.

La citología no tiene un rol en el diagnóstico de $\mathrm{CP}$, dado que hay pocos estudios que la avalen y no existen criterios citológicos que permitan la distinción entre adenoma y carcinoma ${ }^{29,30}$. Se ha utilizado, con buenos resultados, material obteni- do por PAAF para confirmar recurrencia o persistencia de $\mathrm{CP}^{31}$. Sin embargo, es un procedimiento riesgoso, ya que están descritos casos de implantes de carcinoma en el trayecto de la punción ${ }^{29,30}$.

\section{Tratamiento médico}

El manejo perioperatorio del CP está enfocado en el control de la hipercalcemia, dado que esta se asocia a morbimortalidad ${ }^{32}$ (Tabla 4 ).

La primera medida es optimizar el volumen intravascular con solución salina isotónica ${ }^{33}$. La velocidad de infusión depende de las comorbilidades, edad y gravedad de hipercalcemia. Se recomienda un aporte de 3-6 litros las primeras $24 \mathrm{~h}$, para obtener diuresis de $100-150 \mathrm{ml} / \mathrm{h}^{2}$. El uso de diuréticos de asa no está aprobado como medida inicial, dado que produce depleción de volumen intravascular. Solo es recomendable cuando existen síntomas de sobrecarga de volumen ${ }^{33,34}$. Los diuréticos tiazídicos no deben emplearse porque aumentan la reabsorción tubular distal de calcio. La disminución del calcio con estas medidas es efectiva para lograr normocalcemia en $30 \%$ de los casos.

Los bifosfonatos endovenosos están indicados en el manejo de hipercalcemia moderada a grave y se usan concomitantes a la volemización, ya que tienen latencia en el inicio de su acción. Los dos fármacos aprobados son pamidronato $(60-90 \mathrm{mg})$ y zoledronato $(4 \mathrm{mg})$. Este último ha demostrado ser superior en el inicio de acción ( 48 h vs $2-6$ días), efectividad (calcemia normal $88 \%$ vs $70 \%$ a 10 días), tiempo de duración del efecto (32 vs 18 días) y rapidez de la infusión (15 min vs 2 h).

Tabla 4. Manejo de hipercalcemia aguda

\begin{tabular}{|lllcl|}
\hline Tratamiento & Mecanismo de acción & Dosis & Inicio de acción & Duración de efecto \\
$\begin{array}{l}\text { Solución } \\
\text { fisiológica }\end{array}$ & $\begin{array}{l}\text { Volemización y excreción } \\
\text { de calcio }\end{array}$ & & $<6 \mathrm{~h}$ & Durante el tratamiento \\
$\begin{array}{l}\text { Bifosfonatos } \\
\text { endovenosos }\end{array}$ & Inhibidor de resorción ósea & $\begin{array}{l}\text { Zoledronato } 4 \mathrm{mg} \\
\text { Pamidronato } 90 \mathrm{mg}\end{array}$ & $24-72 \mathrm{~h}$ & $1 \mathrm{mes}$ aprox \\
$\begin{array}{l}\text { Denosumab } \\
\text { Cinacalcet }\end{array}$ & $\begin{array}{l}\text { Aghibidor de resorción ósea } \\
\text { de calcio (calcimimético) }\end{array}$ & $120 \mathrm{mg} \mathrm{sc}$ & $4-6$ días & 3 meses \\
\hline Diálisis & Baño pobre en calcio & & $2-3$ días & Titular cada 4 semanas \\
\hline
\end{tabular}




\section{Tabla 5. Clasificación TNM propuesta por la American Joint Committee on Cancer (AJCC VIII)}

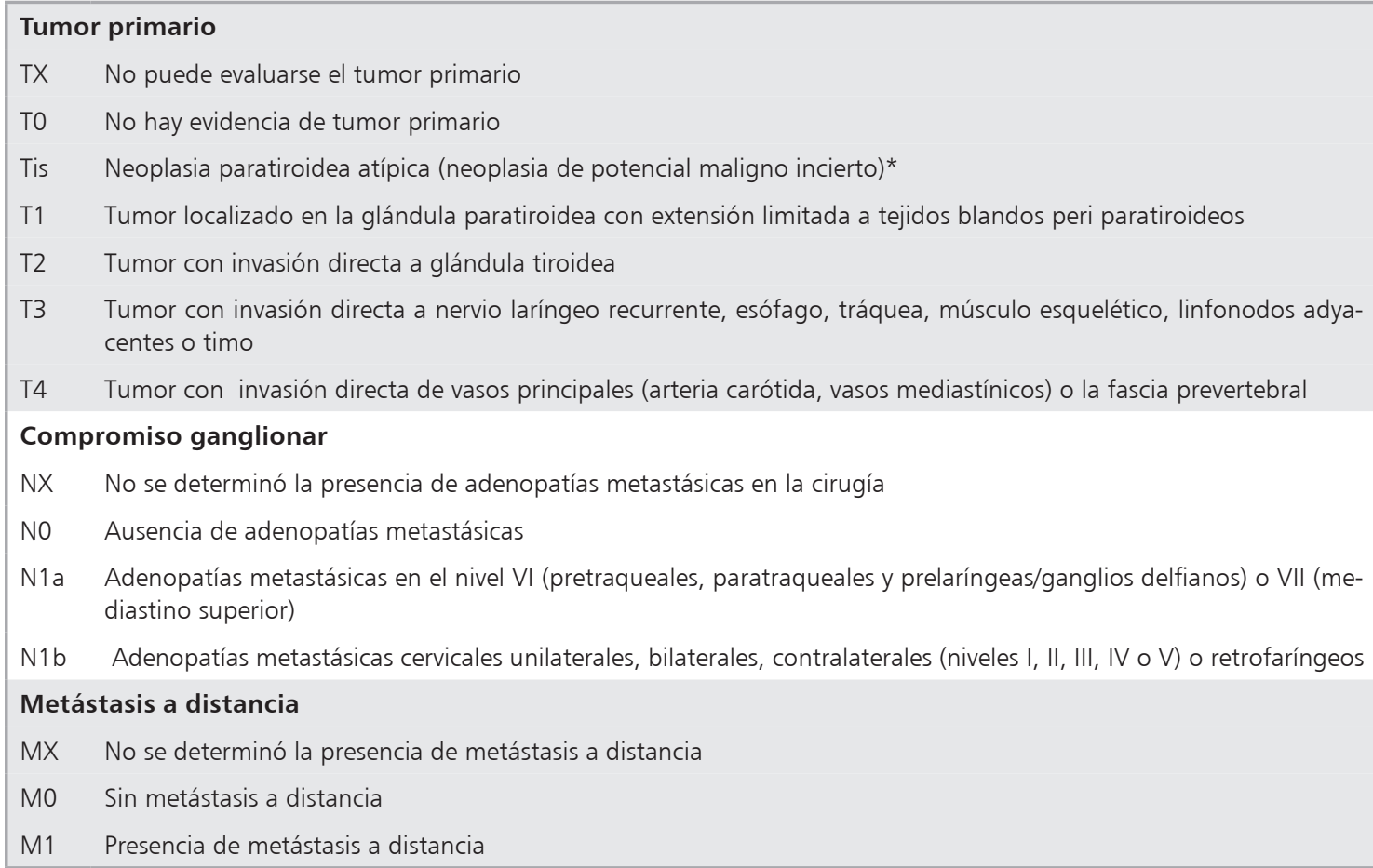

*Neoplasia paratiroidea atípica: para los casos en que no se encuentran todos los hallazgos clínicos o histológicos para ser diagnosticados con riesgo de malignidad (fibrosis, mitosis, necrosis, etc) y sin datos concluyentes (invasión, metástasis) para carcinoma. Ver Anatomía patológica.

Ambos pueden producir una reacción de fase aguda en $10-30 \%$ de los pacientes y están contraindicados en pacientes con clearance de creatinina inferior a $35 \mathrm{ml} / \mathrm{min}$.

El denosumab se utiliza para el tratamiento de hipercalcemia tumoral en pacientes refractarios a bifosfonatos o con contraindicación a ellos. Se sugiere iniciar $120 \mathrm{mg}$ subcutáneo semanal hasta controlar la calcemia y luego mensual.

La diálisis se reserva para calcemias $>18 \mathrm{mg} / \mathrm{dl}$, compromiso neurológico o falla renal aguda ${ }^{35,36}$.

En aquellos pacientes con cirugía no curativa o con contraindicación quirúrgica es importante el tratamiento crónico de la hipercalcemia ${ }^{32}$. Se debe evitar cualquier situación que pueda exacerbarla (uso de litio o tiazidas, hipovolemia, reposo prolongado e ingesta de calcio mayor a $1 \mathrm{~g} /$ día).

Los calcimiméticos, como cinacalcet, son moduladores alostéricos del receptor sensor del calcio, actúan aumentando la sensibilidad del receptor a los niveles circulantes de calcio con lo cual se inhibe la secreción de PTH y secundariamente la calcemia ${ }^{37,30}$. Su inicio de acción es de 2 a 3 días. La dosis es $30 \mathrm{mg}$ cada $12 \mathrm{~h}$ y se titula cada 2-4 semanas, según evolución de calcemia y tolerancia, dado que produce náuseas, vómitos y cefalea $^{37,38}$.

Los bifosfonatos intravenosos pueden repetirse una vez al mes, según evolución de la calcemia, pero su efectividad disminuye con el tiempo ${ }^{39}$. Denosumab puede ser usado crónicamente en casos refractarios a bifosfonatos o a cinacalcet ${ }^{40}$. Es más duradero y no se ajusta por función renal y no parece disminuir efectividad con el tiempo, pero aún la experiencia es escasa.

\section{Manejo quirúrgico de los pacientes con cáncer de paratiroides}

El único tratamiento curativo es la resección quirúrgica completa y se logra con la resección 
oncológica en bloc del tumor primario, evitando la ruptura capsular y asegurando márgenes libres de tumor al momento de la primera cirugía ${ }^{41,42}$. Por esto es fundamental contar previamente con estudios de imágenes para una detallada información anatómica (ecografía, TAC o RM). La resección en bloc consiste en la extirpación completa del tumor junto al lóbulo tiroideo ipsilateral, istmo, músculos pretiroideos comprometidos y disección cervical ganglionar central ${ }^{43}$. La disección radical de cuello modificada y la paratiroidectomía simple no están recomendadas.

La sospecha preoperatoria de $\mathrm{CP}$, el reconocimiento intraoperatorio y la experiencia del cirujano son relevantes para lograr un manejo quirúrgico inicial adecuado. Muchos casos son diagnosticados después de la cirugía y, por lo tanto, son sometidos a procedimientos quirúrgicos iniciales más simples ${ }^{41}$. La resección incompleta tiene el doble de riesgo de recurrencia, pero cualquier resección quirúrgica independiente de su extensión reduce del riesgo de muerte ${ }^{41}$. En casos de cirugía incompleta, se sugiere completarla con una resección en bloc aproximadamente un mes después, con el fin de disminuir las recurrencias ${ }^{44}$, especialmente en los casos con invasión capsular, vascular, perineural o persistencia de PTH y calcio elevado, si se ha descartado enfermedad a distancia. En pacientes portadores de mutaciones germinales CDC73 o MEN1 que desarrollan un CP, se sugiere el manejo estándar de exploración cervical bilateral y resección en bloc del CP identificado ${ }^{44}$.

La información disponible relacionada con la disección cervical central linfonodal es retrospectiva y demuestra una baja tasa de metástasis regionales ${ }^{43}$. Dado que no parece mejorar la sobrevida ni disminuir el riesgo de recurrencia, no se recomienda si no hay sospecha de enfermedad regional, aunque pudiera considerarse en tumores $>3 \mathrm{~cm}$, debido a su probable mayor riesgo de enfermedad metastásica regional ${ }^{44}$.

\section{Tratamiento de recurrencia y metástasis}

Las recurrencias ocurren en hasta 50\% de los pacientes en seguimiento a 3-4 años. La mayoría son locorregionales y en estos casos se recomienda cirugía de los tumores, tanto cervicales como mediastínicos, para disminuir la masa tumoral. En caso de enfermedad metastásica localizada, se recomienda metastasectomía para control de hipercalcemia $^{44}$. En general, el CP es radiorresistente.
Si bien hay reportes que muestran resultados positivos al aplicar radioterapia en el lecho después de la cirugía inicial con disminución de recurrencias y mayor sobrevida libre de enfermedad, un estudio del Cancer Data Base mostró que la radioterapia externa no aumenta la sobrevida ${ }^{41}$. La decisión de usar radioterapia adyuvante debe tomarse con un equipo multidisciplinario.

La quimioterapia no ha mostrado eficacia para el tratamiento del CP. La evidencia con citotóxicos se limita a reportes de casos en los que se usaron algunos esquemas como: dacarbazina sola o asociada a fluorouracilo y ciclofosfamida o bien, metotrexato, doxorrubicina, ciclofosfamida y lomustina ${ }^{4-46}$.

El advenimiento de nuevas terapias moleculares e inmunes podrían mejorar la respuesta a tratamiento del CP. Se ha comunicado que la inmunoterapia anti-PTH podría disminuir la masa tumoral y mejorar hipercalcemia refractaria. Además, antiangiogénicos como sorafenib han mostrado controlar la enfermedad metastásica pulmonar en casos aislados ${ }^{44}$.

La sobrevida global del cáncer paratiroideo es de 14 años, con una tasa de sobrevida a 5 años entre 50 y $85 \%$. El diagnóstico precoz y resección completa del tumor al momento de la cirugía inicial confieren el mejor pronóstico ${ }^{41}$. Se consideran factores clínicos de mal pronóstico y mayor riesgo de recurrencias la edad mayor de 65 años, calcemia inicial mayor de $15 \mathrm{mg} / \mathrm{dl}$, invasión vascular, ,ausencia de parafibromina en el tumor y la presencia de la mutación germinal de CDC73.

El objetivo del seguimiento es la detección precoz de recurrencias locorregionales y de metástasis. Una vez demostrada la normalización de la calcemia, los pacientes deben ser seguidos con calcemia y PTH bianual durante 5 años, luego anualmente. Además, debe realizarse una ecografía cervical al menos una vez al $\mathrm{año}^{47}$. En el caso de cirugías incompletas o pacientes con factores de mal pronóstico, se recomienda control de calcemia y PTH cada 3 meses los primeros 3 años, bianual hasta 5 años y anual posterior ${ }^{44}$. La mayoría de las recurrencia ocurre entre 2,5 y 5 años después de la cirugía inicial. El 25\% desarrolla metástasis a distancia durante el seguimiento. Los sitios más frecuentes son pulmones, huesos e hígado. La mortalidad está dada principalmente por la hipercalcemia intratable que lleva a daño de órgano blanco $^{48,49}$ (Figura 1). 


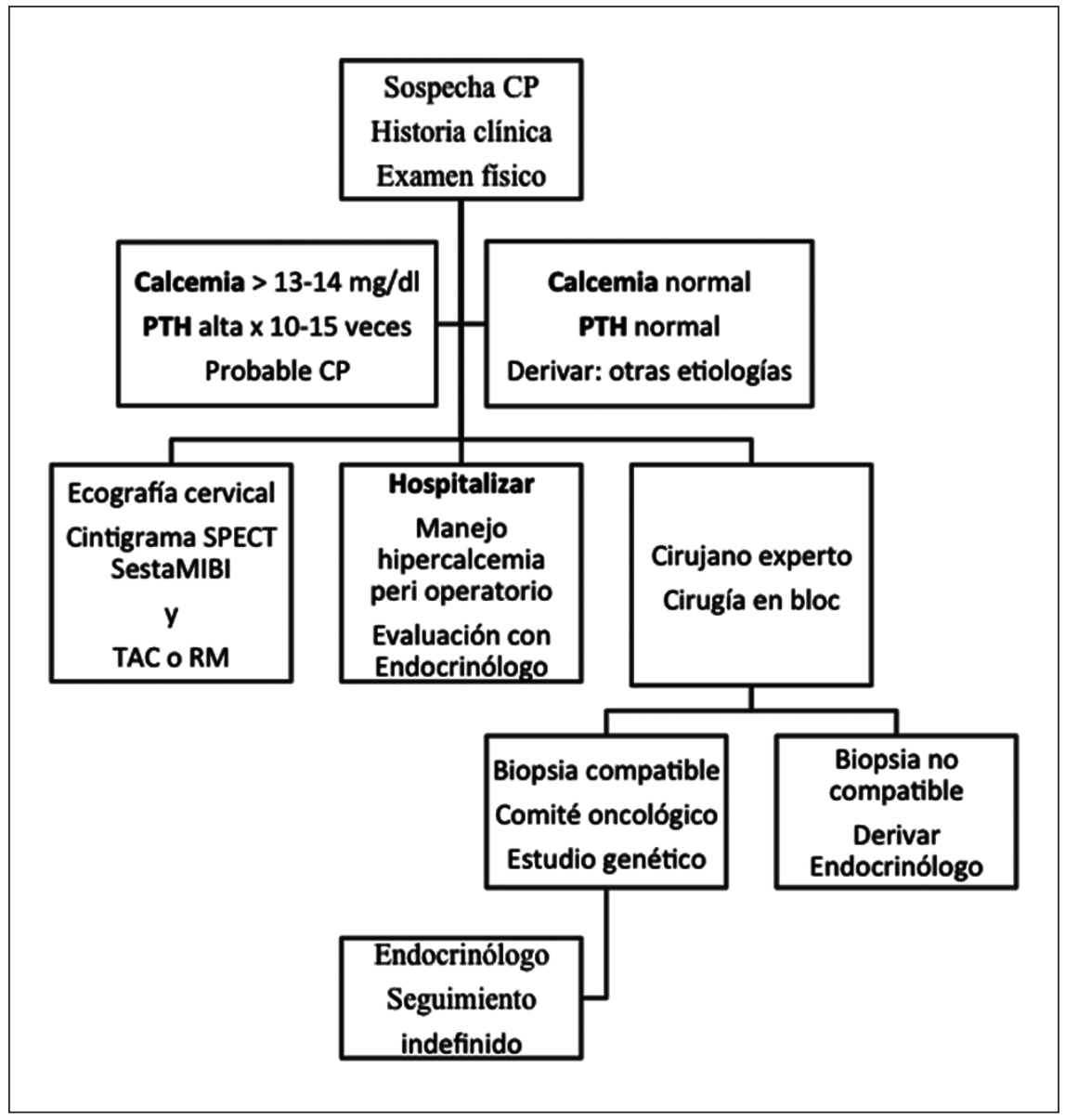

Figura 1. Algoritmo de sospecha, diagnóstico y tratamiento de carcinoma paratiroideo.

\section{Referencias}

1. Kai D, Özgür M. Parathyroid Carcinoma: Diagnosis and Clinical Implications. Turk Patoloji Derg 2015; 31 (Suppl): 80-97.

2. Mohebati A, Shaha A, Shah J. Parathyroid Carcinoma Challenges in Diagnosis and Treatment. Hematol Oncol Clin N Am 2012; 26: 1221-38.

3. Schaapveld M, Jorna FH, Aben KK. Incidence and prognosis of parathyroid gland carcinoma: a population-based study in The Netherlands estimating the preoperative diagnosis. Am J Surg 2011; 202 (5): 590-74.

4. Cetani F, Pardi E, Marcocci C. Update on parathyroid Carcinoma. J Endocrinol Invest 2016; 39: 595-606.

5. Cardoso L, Stevenson M, Thakker RV. Molecular genetics of syndromic and non-syndromic forms of parathyroid carcinoma. Hum Mutat. 2017; 38 (12): 1621-48.

6. Shattuck TM, Valimaki S, Obara T, Gaz RD, Clark OH,
Shoback D, et al. Somatic and germ-line mutations of the HRPT2 gene in sporadic parathyroid carcinoma. NEJM 2003; 349 (18):1722-9.

7. Salcuni A, Cetani F, Guarnieri V, Nicastro V, Romagnoli E, de Martino D, et al. Parathyroid carcinoma. Best Pract Res Clin Endocrinol Metab. 2018; 32: 877-89.

8. Guan B, Welch JM, Sapp JC, Ling H, Li Y, Johnston JJ, et al. GCM2-Activating Mutations in Familial Isolated Hyperparathyroidism. Am J Hum Genet 2016; 99 (5): 1034-44.

9. Marx SJ, Goltzman D. Evolution of Our Understanding of the Hyperparathyroid Syndromes: Historical Perspective. J Bone Miner Res 2019; 34 (1): 22-37.

10. Hara H, Igarashi A, Yano Y, Yashiro T, Ueno E, Aiyoshi $\mathrm{Y}$, et al. Ultrasonographic features of parathyroid carcinoma. Endocr J 2001; 48 (2): 213-7.

11. Christakis I, Vu T, Chuang HH, Fellman B, Figueroa AMS, Williams MD, et al. The diagnostic accuracy of 
neck ultrasound, 4D-Computed tomographyand sestamibi imaging in parathyroid carcinoma. Eur J Radiol 2017; 95: 82-8.

12. Daniela Betea, Iulia Potorac, Albert Beckers. Parathyroid carcinoma: challenges in diagnosis and treatment. Annales d'Endocrinologie 2015; 76: 169-77.

13. Wei $\mathrm{CH}$, Harari A. Parathyroid Carcinoma: Uptodate and Guidelines for Management. Curr Treat Options Oncol 2012; 13(1): 11-23.

14. Givi B, Shah JP. Parathyroid carcinoma. Clin Oncol (R Coll Radiol). 2010; 22 (6): 498-507.

15. El-Hajj Fuleihan G, Arnold A, Drezner MK, Mulder JE. Parathyroid carcinoma. Up to Date. 2019. Disponible en: www. uptodate.com [Consultado el 18 de enero de 2019].

16. Kluijfhout W, Pasternak J, Drake F, Beninato T, Gosnell J, Shen W, et al. Use of PET tracers for parathyroid localization: a systematic review and meta-analysis. Langenbeck's Archives of Surgery 2016; 401 (7): 925-35.

17. Quak E, Blanchard D, Houdu B, Le Roux Y, Ciapuccini $\mathrm{R}$, Lireux B, et al. F18-choline PET/CT guided surgery in primary hyperparathyroidism when ultrasound and MIBI SPECT/CT are negative or inconclusive: the APACH1 study. Eur J Nucl Med Mol Imaging 2018; 45(4): 658-66.

18. Marcocci M, Cianferotti L, Cetani F. Bone disease in primary hyperparathyroidism. Ther Adv Musculoskel Dis 2012; 4: 357-68.

19. World Health Organization. Classification of tumours of endocrine organs. International Agency for Research on Cancer, IARC 2017.

20. Wenig BE. Atlas of Head and Neck Pathology. 3rd Edition Elsevier. 2015.

21. Fletcher C. Diagnostic Histopathology of Tumors, $4^{\text {th }}$ Edition. Churchill Livingstone. 2013.

22. Shattuck TM, Välimäki S, Obara T, Gaz RD, Clark OH, et al. Somatic and germ-line mutations of the HRPT2 gene in sporadic parathyroid carcinoma. N Engl J Med 2003; 349 (18): 1722-9.

23. Howell VM, Haven CJ, Kahnoski K, Khoo SK, Petillo $\mathrm{D}$, Chen J, et al. HRPT2 mutations are associated with malignancy in sporadic parathyroid tumours. J Med Genet 2003; 40 (9): 657-63.

24. Howell VM, Gill A, Clarkson A, Nelson AE, Dunne R, Delbridge LW, et al. Accuracy of combined protein gene product 9.5 and parafibromin markers for immunohistochemical diagnosis of parathyroid carcinoma. J Clin Endocrinol Metab 2009; 94 (2): 434-41.

25. De Lellis, RA. Proliferation markers in neuroendocrine tumors: useful or useless? A critical reappraisal. Verh Dtsch Ges Pathol 1997; 81: 53-61.
26. Farnebo F, Auer, G, Farnebo L, et al. Evaluation of retinoblastoma and Ki-67 immunostaining as diagnostic markers of benign and malignant parathyroid disease. World J Surg 1999; 23: 68-74.

27. DeLellis, RA. Parathyroid tumors and related disorders. Mod Pathol 2011; 24 (Suppl 2): S78-S93.

28. Silva-Figueroa AM, Bassett R Jr, Christakis I, Moreno P, Clarke CN, Busaidy NL, et al. Using a Novel Diagnostic Nomogram to Differentiate Malignant from Benign Parathyroid Neoplasms. Endocr Pathol 2019; 30: 285-96.

29. Agarwal G, Dhingra S, Mishra SK, Krishnani N. Implantation of parathyroid carcinoma along fine needle aspiration track. Langenbecks Arch Surg 2006; 391 (6): 623-6.

30. Alwaheeb S, Rambaldini G, Boerner S, Coiré C, Fiser J, Asa SL. Worrisome histologic alterations following fine-needle aspiration of the parathyroid. J Clin Pathol 2006; 59 (10): 1094-6.

31. MacFarlane MP, Fraker DL, Shawker TH, Norton JA, Doppman JL, Chang RA, et al. Use of preoperative fine-needle aspiration in patients undergoing reoperation for primary hyperparathyroidism. Surgery 1994; 116 (6): 959-64; discussion 964-5.

32. Busaidy NL, Jiménez C, Habra MA, et al. Parathyroid carcinoma: a 22-year experience. Head Neck 2004; 26 : 716.

33. Maier J, Levine S. Hypercalcemia in the Intensive Care Unit: A review pathophysiology, diagnostic and modern therapy. J Intensive Care Med 2015; 30 (5): 235-52.

34. LeGrand S, Leskuski D, Zama I. Narrative review: furosemide of hypercalcemia: An unproven yet common practice. Ann Inter Med 2008; 149: 259-63.

35. Salcuni A, Cetani F, Guarnieri V, Nicastro V, Romagnoli E, De Martino D et al. Parathyroid carcinoma. Best Pract Res Clin Endocrinol Metab 2018; 32 (6): 877-89.

36. Adhikaree J, Nemby Y, Sundar S. Denosumab should be the treatment of choice for bisphosphonate recfractory hypercalcemia of malignancy. BJM Case Rep 2014; 1-5.

37. Collins M, Skarulis C, Bilezikian J, Silverberg S, Spiegel A, Marx S. Treatment of hypercalcemia secondary to parathyroid carcinoma with a novel calcimimetic agent. J Clin Endocrinol Metab 1998; 83 (4): 1083-8.

38. Silverberg S, Rubin M, Faiman C, Peacock M, Shoback $\mathrm{D}$, Smallridge $\mathrm{R}$, et al. Cinacalcet hydrochloride reduces the serum calcium concentration in inoperable parathyroid carcinoma. J Clin Endocrinol Metab 2007; 92(10): 3803-8.

39. Mohebati A, Shaha A, Shah J. Parathyroid carcinoma, challenges in diagnostic and treatment. Hematol Oncol Clin N Am 2012; 26: 1221-38.

40. Vellanki P, Langue K, Elaraj D, Koop P, El Muayed M. 
Denosumab for management of parathyroid carcinoma - mediated hypercalcemia. J Clin Endocrinol Metab 2014; 99(2): 387-90.

41. Asare EA, Sturgeon C, Winchester DJ, Liu L, Palis B, Perrier N, et al. Parathyroid Carcinoma: An Update on Treatment Outcomes and Prognostic Factors from the National Cancer Data Base (NCDB). Ann Surg Oncol 2015; 22 (12): 3990-5.

42. Schulte KM, Talat N, Galata G, Gilbert J, Miell J, Hofbauer LC, et al. Oncologic resection achieving r0 margins improves disease-free survival in parathyroid cancer. Ann Surg Oncol 2014; 21 (6): 1891-7.

43. Randle RW, Schneider DF. Lymph Node Dissection Versus No Lymph Node Dissection for Parathyroid Cancer. In: Angelos P, Grogan R, Editors: Difficult Decisions in Surgery: An Evidence-Based Approach. Springer, Cham, 2018. pp. 193-208.

44. Rodrigo J, Hernández-Prera J, Randolph G, Zafereo M, Hartl D, Silver C. Parathyroid cancer: An update. Cancer Treatment Reviews. 2020; 86:1-1045. Hsu KT, Sippel RS, Chen H, Schneider DF. Is central lymph node dissection necessary for parathyroid carcinoma? Surgery 2014; 156 (6): 1336-41.

45. Wei $\mathrm{CH}$, Harari A. Parathyroid carcinoma: update and guidelines for management. Curr Treat Options Oncol 2012; 13 (1): 11-23.

46. Storvall S, Ryhanen E, Bensch F, Heiskanen I, Kytola S, Ebeling T, Makela S. RecurrentMetastasized Parathyroid Carcinoma-Long-Term Remission After Combined Treatments With Surgery, Radiotherapy, Cinacalcet, Zoledronic Acid, and Temozolomide. JBMR Plus 2018; 3 (4): e10114.

47. Salcuni AS, Cetani F, Guarnieri V, Nicastro V, Romagnoli E, de Martino D, et al. Parathyroid carcinoma. Best Pract Res Clin Endocrinol Metab 2018; 32 (6): 877-89.

48. Wei $\mathrm{CH}$, Harari A. Parathyroid carcinoma: update and guidelines for management. Curr Treat Options Oncol 2012; 13 (1): 11-23.

49. Ferraro V, Sgaramella L, Di Meo G, Paolo F, Logoluso F. Current concepts in parathyroid carcinoma. BMC Endocrine Disorders 2019; 19: 46-55. 